\title{
The comfort and safety of a novel rolling mechanical indentation device for the measurement of lumbar trunk stiffness in young adults
}

Benjamin T. Brown ${ }^{1 *}$, Alexandra Blacke ${ }^{1}$, Vanessa Carroll1, Petra L. Graham², Greg Kawchuk ${ }^{3}$, Aron Downie ${ }^{1}$ and Michael Swain ${ }^{1}$

\begin{abstract}
Background: The measurement of Posterior-Anterior (P-A) spinal stiffness is a common component of the physical examination of patients presenting with spinal disorders. The aim of this assessment is to provoke pain and/or to determine the degree of resistance or compliance of these structures and the associated soft-tissues to loading. This information, combined with other patient-specific history and examination findings, is integrated into the clinical reasoning process and is used to guide treatment decisions. Unfortunately, there are inter-rater reliability and standardisation issues associated with the manual performance of this type of assessment. In an attempt to remedy these issues researchers have developed mechanical devices for the measurement of spinal stiffness. The aim of this research is to investigate the comfort and safety of a novel device for measuring P-A trunk stiffness in a sample of young adults.
\end{abstract}

Methods: A sample of young adults from a general population was recruited in May 2016 from Sydney, Australia. Demographic, anthropometric and clinical variables were collected prior to participants undergoing a lumbar P-A trunk stiffness assessment involving a mechanical indentation device called the VerteTrack. The primary outcomes for the study were key feasibility items; overall assessment time, perceived comfort measured both during and after the procedure, and adverse events. Univariate ordinal logistic regression was used to identify key variables associated with a participant's subjective report of comfort both during and after the VerteTrack assessment.

Results: Eighty four participants (35\% female) with a median age of 23 years (IQR $=3$ ) took part in the research. The mean assessment time for the Vertetrack assessment was $11.6 \mathrm{~min}(\mathrm{SD}=2.1)$. Increasing load $(p<0.001)$ and increasing number of days with lower back pain $(p=0.009)$ were associated with decreased comfort ratings during the procedure. The vast majority 63/84 (75\%) of participants rated the overall assessment experience as comfortable. There were two minor, short-lived adverse events recorded leading to an adverse event rate of $2.4 \%$ $(2 / 84)$.

Conclusions: The results of this study suggest that the VerteTrack device is well-tolerated and can be used safely and efficiently when measuring P-A stiffness of the lumbar trunk in young adults.

Trial registration: Not applicable.

Keywords: Low back pain, Spine, Trunk, Stiffness, Adult

\footnotetext{
* Correspondence: benjamin.brown@mq.edu.au

'Department of Chiropractic, Macquarie University, Balaclava Rd, North Ryde, NSW 2109, Australia

Full list of author information is available at the end of the article
} 


\section{Background}

Clinicians who use manual therapy use a variety of methods to examine patients presenting with spinal disorders including patient interview, special tests and physical exam. With these tools, a clinician seeks to collect and assemble all relevant clinical data for the purposes of forming a working diagnosis, prognosis and management plan for their patient [1-3].

One aspect of physical examination commonly used by clinicians is the assessment of spinal posterior to anterior (P-A) stiffness. Traditionally, P-A load/s of between 30 and 200 Newtons [4] (N) are manually applied to the spinous process of a target vertebra or group of vertebrae with the patient in a prone or seated position. The clinician then compares this subjective measurement of stiffness to those taken in adjacent areas of the patient's spine. Simultaneously, the clinician also makes comparisons with experiential data from previous patient states/encounters as well as experiential data from their clinical training to determine 'normal' and 'abnormal' responses to the test procedure [3]. The aim of this assessment is to provoke pain and/or to determine the degree of resistance or compliance of these structures and the associated soft-tissues to loading [5]. This information, combined with other patient-specific history and examination findings, is integrated into the clinical reasoning process and is used to guide treatment decisions. The assessment of spinal P-A stiffness can also be used as a post-treatment outcome $[2,6]$.

Unfortunately, researchers have highlighted problems with the manual performance and interpretation of this type of examination; specifically poor inter-rater reliability $[1,7,8]$, and a lack of standardisation of both the testing procedure and the scales used to quantify the stiffness ratings [1]. Nicholson et al. [5] also highlighted that low palpation sensitivity and limited subjective perception may also contribute to the poor reliability during stiffness assessments of viscoelastic structures such as the spine. Furthermore, clinicians do not use consistent forces when manually assessing spinal stiffness which can complicate the interpretation of the information being perceived [2]. This has led researchers to develop mechanical indentation devices that mimic this style of examination and provide a standardised and reliable measure of this clinical construct [9-15]. The majority of the devices described in the literature involve a motorised indenter head mounted on a gantry that applies a P-A load to a single spinal segment. Typically a series of increasing loads (measured in Newtons) are applied to the target segment, with measurement of deformation $(\mathrm{mm})$ used as a proxy for movement in the target segment and the associated tissues. A modulus of stiffness $(\mathrm{N} / \mathrm{mm})$ is then calculated for that spinal level. One of the challenges associated with previous devices is that in order to measure the stiffness of multiple spinal segments (e.g. lumbar spine) the device must be repositioned and recalibrated at each segment, a cumbersome and time consuming procedure for all involved. In an attempt to streamline the automated measurement of spinal stiffness, a novel device called the Vertetrack has been developed. The device, which is currently used for research purposes, features a rolling indenter head capable of measuring the combined P-A stiffness of the spine and the adjacent tissues (trunk stiffness) without the need for repositioning and recalibration between segments/regions of the spine.

The aim of this study was to investigate the comfort and safety of the Vertetrack, a research tool for measuring P-A lumbar trunk stiffness in a sample of young adults. This information, when combined with data on accuracy and reliability, will help to determine the suitability of this device for use in research and clinical settings.

\section{Methods \\ Design \\ Cross-sectional study.}

\section{Ethics approval}

Ethics approval was obtained from the Macquarie University human ethics committee - Reference number: 5201600008 .

\section{Sampling}

A convenience sample of young adults were recruited from the student population at Macquarie University, Australia. The research was conducted within a dedicated laboratory space.

\section{Eligibility criteria}

Participants who were $\geq 18$ years of age were eligible to participate in the study. Due to the nature of the assessment procedure, participants were excluded if they were: unable to lie in the prone position for $\geq 20 \mathrm{~min}$; were in the second or third trimester of pregnancy; were unable to maintain their breathing cycle in passive expiration (Functional residual capacity) for at least $10 \mathrm{~s}$; or had recently undergone head, neck, thoraco-abdominal or spinal surgery. The presence or absence of spinal pain was not included as part of the eligibility criteria.

\section{Outcome measures}

All participants were issued with an information and consent form. Consenting participants then completed a baseline questionnaire which contained questions regarding demographic (age, gender, ethnicity) and clinical variables (low back pain frequency in the past 7 days, and smoking status). With reference to low back pain 
frequency, participants were asked "How many days did you have low back pain during the last week?" with the answer recorded on a scale from zero to seven. The data pertaining to smoking status was sorted into three categories: never smoked, used to smoke but have now quit (past smoker), and current smoker.

Upon completion of the baseline questionnaire, participants underwent a physical examination designed to capture relevant anthropometric characteristics: seated height $(\mathrm{cm})$, standing height $(\mathrm{cm})$, weight $(\mathrm{kg})$, waist P-A diameter and circumference $(\mathrm{cm})$, and chest P-A diameter and circumference $(\mathrm{cm})$. Standing height and weight measurements were converted into body mass index (BMI) units $\left(\mathrm{kg} / \mathrm{m}^{2}\right)$. The primary outcomes for the study were: lumbar P-A trunk stiffness assessment: overall assessment time, perceived comfort, adverse events and mechanical downtime. Time was measured in minutes, and comfort level was measured on a 7-point bipolar-scale adapted from the work of Hernandez et al. [16]; 0 = strong discomfort, 1 = moderate discomfort, $2=$ mild discomfort, $3=$ neutral, $4=$ mild comfort, $5=$ moderate comfort, and $6=$ strong comfort. Perceived comfort was rated by the participant both during the assessment and also at the completion of the assessment. Adverse events were recorded by the research assistant. Participants were also asked to describe their experience of the VerteTrack device at the end of the assessment.

\section{VerteTrack device}

The VerteTrack device was used to measure the lumbar P-A trunk stiffness of participants. The VerteTrack device functions to apply a pre-selected vertical load continuously over a specific spinal region (e.g. lumbar spine). The device consists of a solid, cube-shaped aluminium gantry (Width $1080 \mathrm{~mm} \times$ Height $1090 \mathrm{~mm} \times$ Length $1510 \mathrm{~mm}$ ), on lockable casters, that can be positioned over a participant lying in the prone position on a standard padded-plinth (Fig. 1). The frame

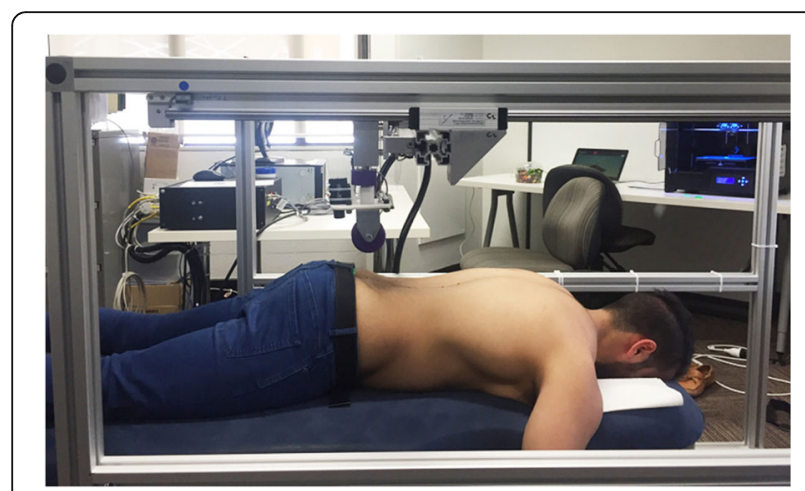

Fig. 1 The VerteTrack device positioned over a patient on a standard plinth is used to provide a rigid support for the indenter apparatus which applies a vertical load to the region of interest and houses a sensor to measure the resulting tissue deformation. The indenter apparatus consists of a rod suspended within a linear bearing to permit nearfrictionless vertical translation and an indentation roller comprising two circular plastic disks (Diameter $70 \mathrm{~mm}$, width $15 \mathrm{~mm}$ ). Force transfer to the test subject is via the rod loaded with masses of increasing magnitude applied through the indentation roller. These straddle the test subject's spinous processes thus providing a rolling contact point for the application of P-A loads (Fig. 2).

The indentation roller is moved in the $\mathrm{X}$ (longitudinal, superior-inferior), $\mathrm{Y}$ (transverse, left-right) and $\mathrm{Z}$ (vertical, P-A) axes by a stepping motor system (Resolution $=0.007 \mathrm{~mm}$ ) (Stepperonline.com, China). The vertical position of the indenter relative to the frame is measured by a string potentiometer which provides realtime feedback to the control system (Resolution $=0.020 \mathrm{~mm})($ TE Connectivity, USA). Control of all motors and acquisition of the potentiometer sensor signal is controlled through custom software written in Labview (National Instruments, USA). Using this program, it is possible to position the indenter apparatus at defined way-points along the spine then have the indenter apparatus follow the pre-defined trajectory. The result is a continuous and real-time quantification of the bulk deformation of any spinal region for a given mass over a defined trajectory. Using a series of increasing masses, the force-deformation profile of the spinal region of interest can then be produced.

As the indenter roller is moved over the lumbar region, the spine and associated soft tissues are loaded by the weight of the indenter unit which can be increased or decreased by increments of $10 \mathrm{~N}$ (Maximum $=60 \mathrm{~N}$ ) using weight plates. Movement of the indentation roller along a spinal region ( $\mathrm{X}$ and $\mathrm{Y}$ axes) is planned by the

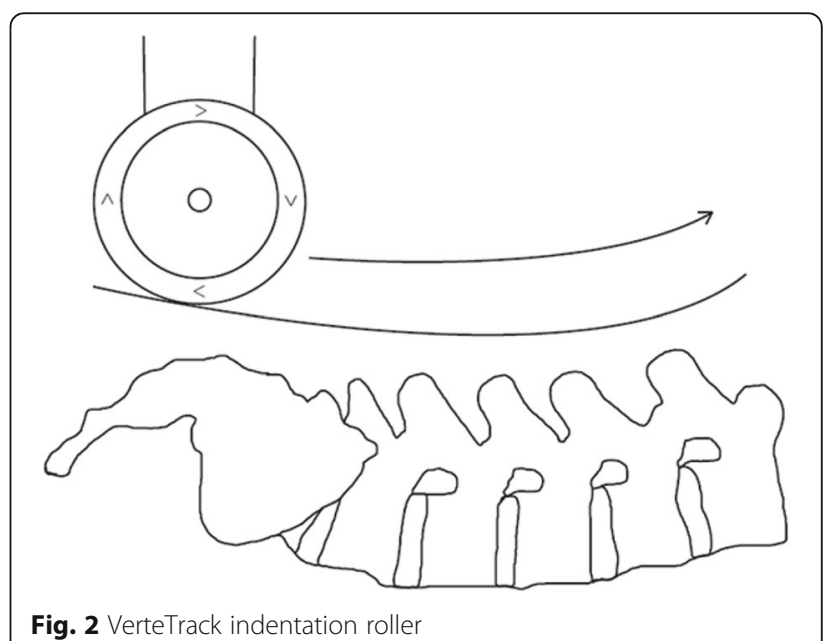


operator who uses a mounted laser to identify waypoints along the area of the trunk to be tested (Typically each spinous process). The indentation roller then follows these points over the bony and soft tissue contours of the patient's lumbar spine, which includes the area between each way-point, through a smooth curvilinear trajectory termed the trace.

During data acquisition, the indentation roller is mechanically lowered onto the participant's trunk. The Z-axis stepping motor is used only to set down then lift off the indenter roller at the beginning and end of a trace respectively. Once the indenter roller has been positioned on the subject's trunk, there is no further contribution to the movement in the negative $\mathrm{Z}$-axis from the stepping motor during a trace. Any P-A movement that is achieved is created via the effect of gravity on the mass of the indenter roller/arm and any additional weight plates. This ensures continual contact between the indenter head and the test subject's trunk and serves to maintain a gravity-constrained rate of loading during the assessment. When testing is complete, the stepping motor system lifts the indenter roller and indenter apparatus from the subject. A video of the VerteTrack in action has been included in Additional file 1 .

\section{Lumbar P-A trunk stiffness assessment}

Participants in this study were required to wear gowns that opened at the back allowing researchers to access their spine and upper sacrum. Participants were asked to lie prone on the plinth. Once a participant was comfortable on the plinth, researchers palpated and marked the spinous processes of the sacrum (landing point), each of the lumbar vertebrae, and the 12th thoracic vertebrae (lift-off point). The Vertetrack gantry was then moved over the participant until the indentation roller was positioned over the landing point, which in this study was the first sacral tubercle. A vertically-orientated laser (GLX Laser Site, Barska) in line with the roller wheel apex was then turned on and then used to align the roller to each marked way-point on the subject's back (i.e. spinous processes). When the laser matched the marks, the position was recorded and a roller trajectory calculated (Fig. 3).

Once the roller trajectory had been established, the roller was lowered onto the landing point. The participant was then instructed to breathe in, then passively expire (functional residual capacity [FRC]). The indenter head was then moved through the pre-calculated roller trajectory by the stepping motor system while the roller itself was free to displace the bony and soft tissues of the lumbar region in the $\mathrm{Z}$-axis $(\sim 10 \mathrm{~s})$. When the indenter head reached the end of the trajectory at the 12th thoracic vertebra, it was raised off the participant and returned to the region just above the landing point. The

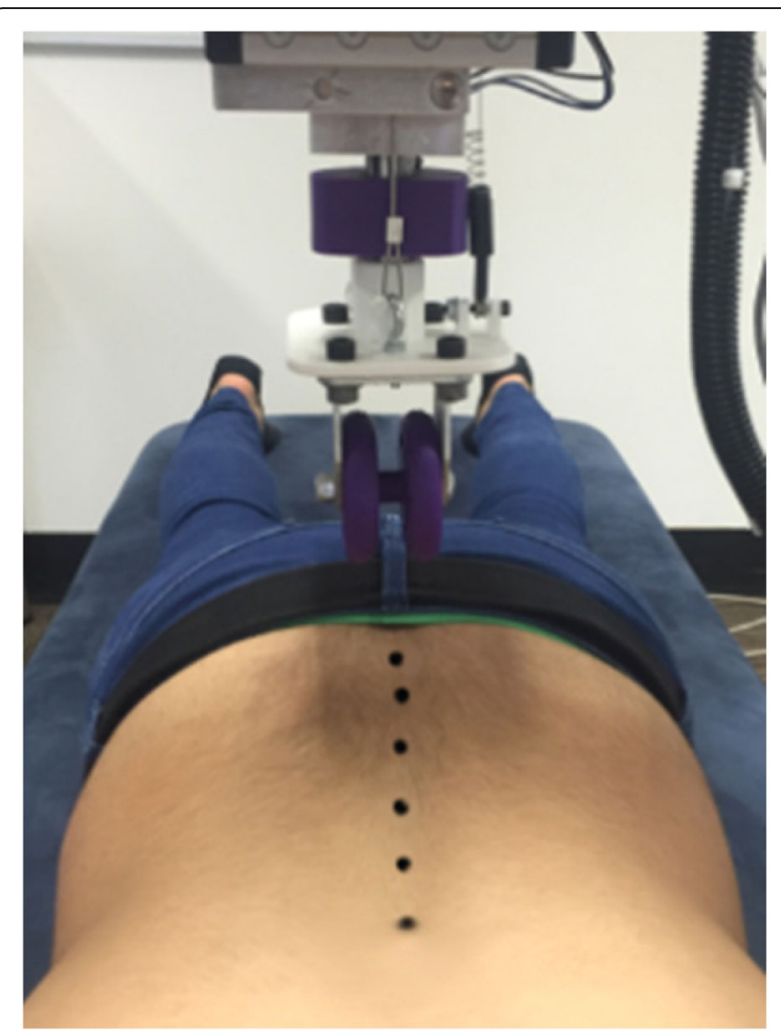

Fig. 3 Bony surface landmarks for the creation of an individualspecific trajectory file

participant was then instructed to breathe normally. The initial trace $(0 \mathrm{~N})$ represents the minimal load configuration which includes the unweighted load receptacle, the indenter arm and the indenter roller. No additional weight is added to this initial trace which is used as the baseline measurement. Successive $10 \mathrm{~N}$ weights were then added to the load receptacle (up to a maximum load of $60 \mathrm{~N}$ ) after each trace, with a rest period of approximately $1 \mathrm{~min}$ between loading cycles. Participants had access to an emergency stop button in the event that they felt discomfort and wished to terminate the assessment. In all, participants received seven traces with incrementally increasing loads $(0 \mathrm{~N}, 10 \mathrm{~N}, 20 \mathrm{~N}, 30 \mathrm{~N}$, $40 \mathrm{~N}, 50 \mathrm{~N}$ and $60 \mathrm{~N}$ ). Data from the traces were compiled and used to calculate the terminal stiffness of the lumbar region. Terminal stiffness is defined as the ratio of the maximal applied force $(60 \mathrm{~N})$ to the maximal resultant displacement $[13,17]$, and was calculated for the palpated spinal levels corresponding to L5, L3 and L1. Localisation of L5 spinous process was achieved using a combination of bony landmark and motion palpation as recommended by Merz et al. [18].

\section{Quantitative analyses}

Data were collated, cleaned, and analysed and descriptive statistics were generated for all variables. The results for 
the descriptive analysis are reported as mean with standard deviation (SD) or median and interquartile range (IQR). Univariate ordinal logistic regression was used to identify any variables correlated with a participant's overall comfort rating. Univariate mixed-effects ordinal logistic regression was then performed to identify independent variables that were correlated with a participant's comfort rating during each loading phase. The mixed-effects model includes a random effect for participant to control for the repeated measures on each participant. The results for the ordinal logistic regression analyses are reported as odds ratios with $95 \%$ confidence intervals and $p$-values. The significance level $(\alpha)$ was set at 0.05 , and all assumptions were checked and determined to have been met unless otherwise reported. All analyses were conducted using the statistical software package R, version 3.1.2 (R Core Team, Vienna, Austria).

\section{Qualitative analyses}

All mechanical and adverse events were described in full. Content analysis [19] was used to determine the major and minor themes in the data relating to the participant's experience of the VerteTrack assessment.

\section{Results}

Eighty four participants ( $35 \%$ female) consented to participate in the research. The age data were right skewed with a median age of 23 years $(\mathrm{IQR}=3)$. With reference to ethnicity, $57 \%(48 / 84)$ were Caucasian, $33 \%(28 / 84)$ were Asian, 1\% (1/84) were Pacific Islander and 7\% (7/ 84) were not stated. The descriptive statistics for anthropometric variables are detailed in Table 1.

With regards to smoking status, $82 \%(69 / 84)$ of participants reported that they had never smoked, $15 \%(13 / 84)$ reported that they used to smoke but have now quit, and $2 \%(2 / 84)$ of the sample were current smokers. A large proportion $(77.3 \%,[65 / 84])$ of the sample population reported one or more days of lower back pain in the week prior to the assessment (Fig. 4). There were several outliers in the assessment time data (Fig. 5) which represented cases where software or equipment did not function as expected (Equipment events). The time taken to reboot software and/or recalibrate the device contributed to these outliers which have been removed from the analysis. Assessment times were normally distributed with a mean assessment time of 11.6 min with a standard deviation of $2.1 \mathrm{~min}$ (Fig. 5).

In an attempt to standardize the stiffness measurements between the VerteTrack and the other devices described in the literature, terminal stiffness measures were calculated for three points in lumbar region - L5, L3, and L1 (Fig. 6A \& B). The mean terminal stiffness at the level of L5 was $1.0 \mathrm{~N} / \mathrm{mm}(\mathrm{SD}=0.15)$, and at the level of L3 was $1.0 \mathrm{~N} / \mathrm{mm}(\mathrm{SD}=0.16)$. The terminal stiffness data for L1 were right skewed with a median of $1.0 \mathrm{~N} / \mathrm{mm}$ (IQR $=0.21)$. All participants received the full $60 \mathrm{~N}$ loading to the lumbar region.

The comfort ratings for the cohort are shown in Fig 7. With respect to overall comfort, the most frequent rating was that of neutral or mild comfort. There were no observations seen for the strong comfort or strong discomfort categories in the overall comfort data. Collapsing the overall comfort data into two categories (comfortable or uncomfortable) highlighted that $75 \%$ of the sample population rated the overall experience as comfortable.

\section{Univariate analyses}

Mixed-effects ordinal logistic regression was used to model the influence of each of the variables age, gender, assessment time, number of days with lower back pain in the past week, BMI, smoking status and load in Newtons on participant comfort ratings during each loading phase. Of these variables, the presence of an increasing number of days with lower back pain in the past week, and increasing load were significantly associated with decreased comfort ratings (Table 2). Ordinal logistic regression was used to model the influence of these same variables (Excluding individual loads) on the overall comfort rating for the assessment. There was no evidence to suggest that any of

Table 1 Descriptive statistics for the anthropometric variables

\begin{tabular}{|c|c|c|c|c|c|}
\hline Variable & Mean & Median & Standard Deviation & Interquartile Range & Range (Min, Max) \\
\hline Sitting height $(\mathrm{cm})$ & 171.6 & 172 & 9.5 & 15.2 & $145.0,190.0$ \\
\hline Standing height $(\mathrm{cm})$ & 90.8 & 91 & 5.4 & 8.5 & $77.0,102.0$ \\
\hline Weight (kg) & 72.0 & 69.4 & 15.8 & 19.1 & $44.1,124.2$ \\
\hline $\mathrm{BMI}\left(\mathrm{kg} / \mathrm{m}^{2}\right)$ & 24.3 & 23.8 & 3.9 & 4.1 & $17.4,37.0$ \\
\hline Waist circumference $(\mathrm{cm})$ & 84.2 & 82.5 & 10.4 & 11.6 & $66.0,118.0$ \\
\hline Chest circumference $(\mathrm{cm})$ & 94.5 & 93.0 & 9.6 & 9.5 & $78.0,127.0$ \\
\hline Waist P-A diameter $(\mathrm{cm})$ & 18.7 & 18.0 & 3.4 & 4.5 & $12.5,28.5$ \\
\hline Chest P-A diameter $(\mathrm{cm})$ & 18.5 & 18.5 & 3.2 & 4.0 & $12.5,27.5$ \\
\hline
\end{tabular}

$P-A$ posterior to anterior, $\mathrm{cm}$ centimetres, $\mathrm{kg}$ kilograms, $\mathrm{m}^{2}$ metres squared, min minimum, max maximum 


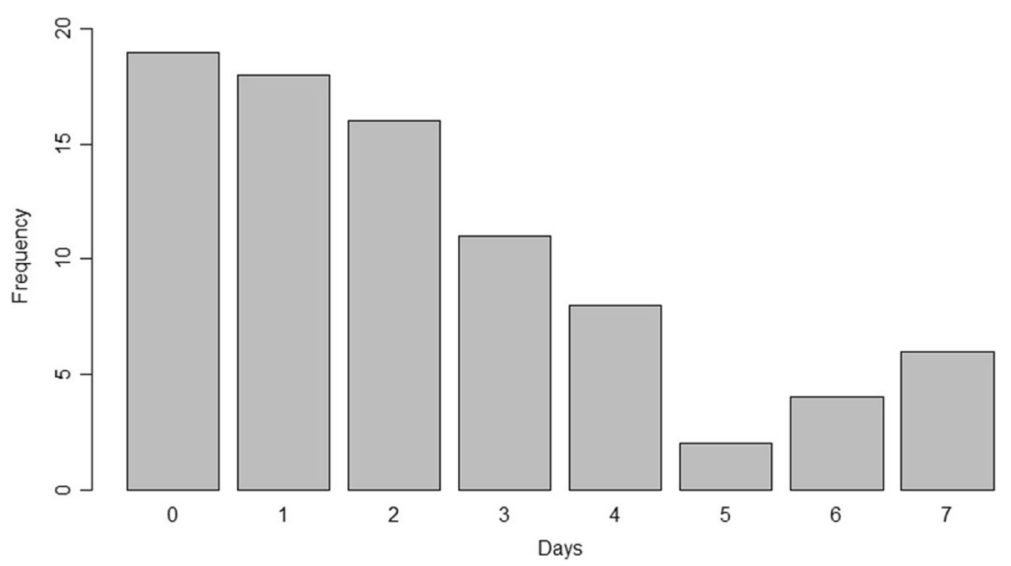

Fig. 4 Barchart of self-reported days with lower back pain in the past week

these variables were associated with the overall comfort rating.

\section{Equipment events}

There were four unexpected equipment-related events. On four occasions, software or equipment did not function as expected during the trunk stiffness assessment. These events required the research assistant to either reboot the software and/or reposition the device before continuing the assessment. No ill-effects were reported by the participants in these cases.

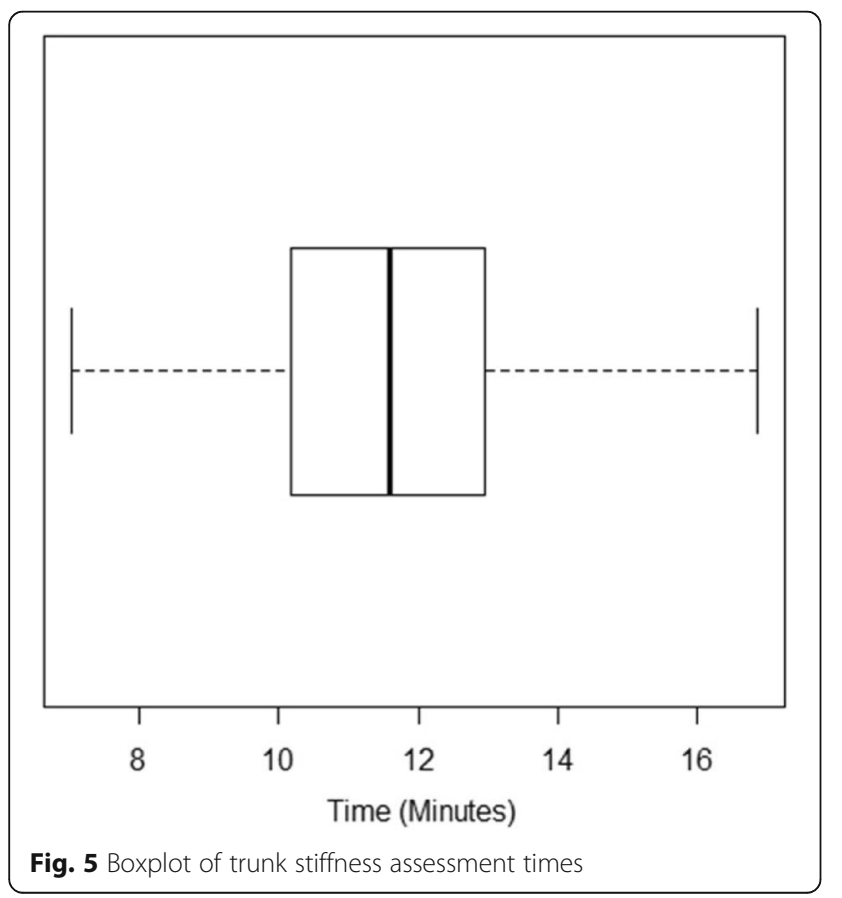

\section{Adverse events}

There were two adverse events reported. One participant reported an aggravation of pre-existing rib pain in the day following the stiffness assessment. Another participant reported aggravation of pre-existing lower back pain resulting in bilateral referred leg pain during the assessment. Of the two participants that made these reports, the reactions were short-lived and neither required remedial treatment. Therefore, the rate of adverse events using the VerteTrack device in this population was $2.4 \%(2 / 84)$. Although some participants reported some discomfort during the stiffness assessment, the emergency stop button was not utilised at any time during this study.

\section{Participant experience}

Content analysis was performed by a single author (BTB) on the data relating to the participant's subjective experience of the VerteTrack assessment. Comments were first sorted into three categories; positive, negative and mixed. Comments that contained both positive and negative remarks, or neutral statements were categorised as mixed. Eighty two participants provided feedback on the VerteTrack assessment. Purely positive comments were made by 29\% (24/82) of the sample, $45 \%(37 / 82)$ gave mixed responses and $24 \%$ (20/82) made negative comment. Participants who made positive comment typically stated that the VerteTrack assessment was comfortable/therapeutic and enjoyed the novel experience. Major themes in the mixed and negative categories were that the indenter roller was perceived as being too rough and/or hard which created a pinching or catching sensation as it rolled up the upper lumbar spine. Participants also commonly reported discomfort as load was increased, particularly in the upper lumbar spine. Minor 

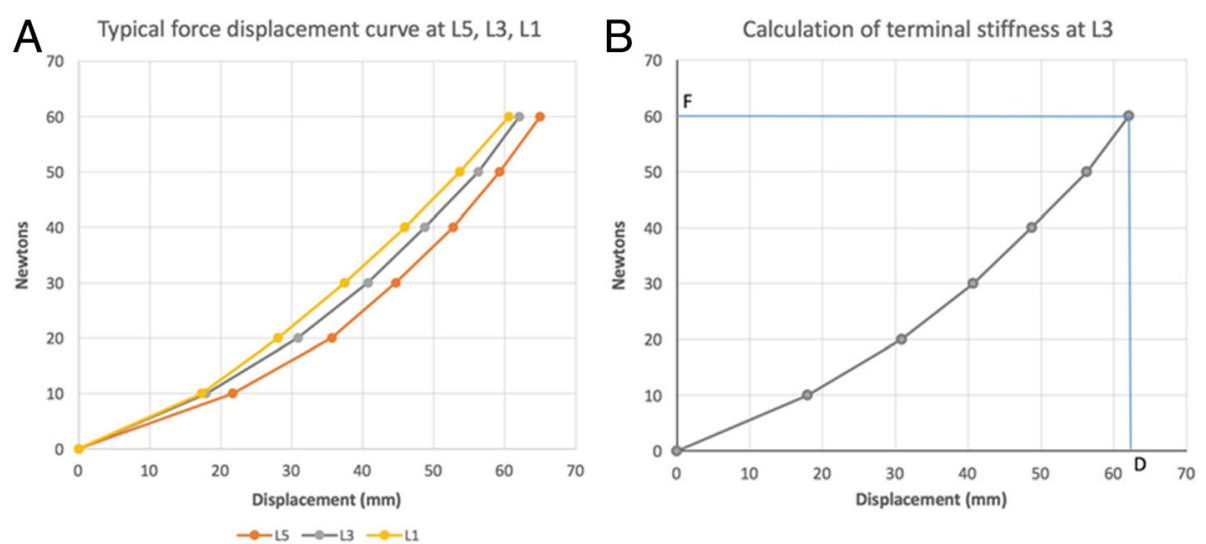

Fig. 6 a Force Displacement Curve. Typical force-displacement curves for levels L5, L3 and L1 (Participant \#43). b Calculation of Terminal Stiffness. Terminal stiffness is calculated by dividing the maximum applied force (F) by the maximal displacement (D)

themes included reports that being in the prone position for the assessment was somewhat uncomfortable, the indenter head reproduced pain, and that the style of the assessment was unusual/foreign.

\section{Discussion}

The aim of this study was to investigate the comfort and safety of the VerteTrack for measuring P-A lumbar trunk stiffness in a sample of young adults.

With regard to safety, the rate of adverse events associated with the use of the VerteTrack device in this population was low (2.4\%). The adverse events that did occur were thought to be related to Vertetrack testing, but this could not be confirmed with the study design. Adverse events attributed to Vertetrack testing were minor and short-lived, and involved two participants with pre-existing neuromusculoskeletal conditions.

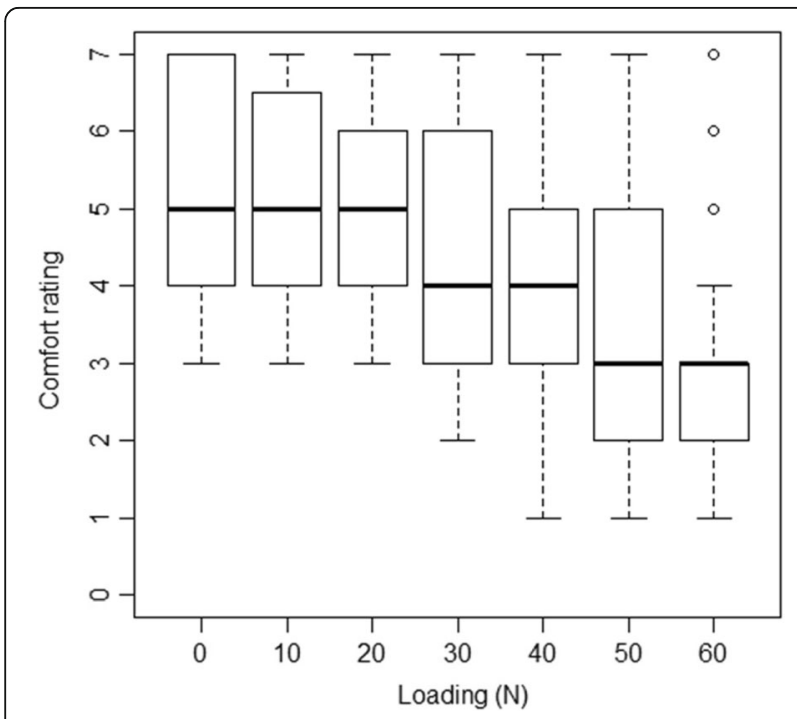

Fig. 7 Boxplots of comfort ratings with incremental loading ${ }^{\circ}=$ Outlier
There is a paucity of data with respect to the safety (specifically harms data) relating to other mechanical indentation devices in the literature. The majority of the work in this area has been conducted on young, asymptomatic populations where adverse events are seldom discussed or reported. Edmondston et al. [12] investigated the effect of body position on the spinal stiffness at L3 and L5 in 12 asymptomatic participants with an average age of 28.8 years. The mechanical device utilised by Edmondston et al. applied loads of between 30 and $80 \mathrm{~N}$, and was equipped with a panic button facility. The researchers stated that no participant had cause to activate the panic button at any time during the research. However, Edmondston et al. also listed "undue pain or discomfort during testing and development of any adverse symptoms that may have been associated with the procedures" as a withdrawal criteria in their methods section. It is therefore unclear whether or not there were participants who were excluded from the final analysis due to adverse reactions. In addition, Latimer et al. [15] developed a mechanical device for measuring spinal stiffness that was capable of delivering force up to $105 \mathrm{~N}$ to a test subject. These researchers reported that since developing their device over 100 test subjects had underwent an assessment with only one of these subjects reporting a minor, short-duration adverse event.

Based on an analysis of the comfort data, both during and after the procedure, it appears that the VerteTrack assessment was considered comfortable by the majority (75\%) of participants in this study. A strong theme that emerged however in both the qualitative and quantitative analyses was that comfort ratings were inversely related to loading i.e. increasing loads resulted in lower comfort ratings. Kumar and Stoll [11] state that loads between 45 and $135 \mathrm{~N}$ allow for reliable measurement of spinal stiffness while still being comfortable. Based on the descriptive statistics, participants found that comfort 
Table 2 Results from the univariate analyses of the comfort data

\begin{tabular}{|c|c|c|c|c|}
\hline \multirow[b]{2}{*}{ Variable [Reference Category] } & \multicolumn{2}{|c|}{ Overall comfort (ordinal logit model) } & \multicolumn{2}{|c|}{ Comfort during the assessment (mixed-effects ordinal logit model) } \\
\hline & Odds ratio $(95 \% \mathrm{Cl})$ & $p$-value & Odds ratio $(95 \% \mathrm{Cl})$ & $p$-value \\
\hline Age & $1.06(0.96,1.18)$ & 0.235 & $1.00(0.90,1.12)$ & 0.975 \\
\hline Assessment time (minutes) & $1.00(0.99,1.00)$ & 0.772 & $1.00(0.92,1.09)$ & 0.939 \\
\hline $\mathrm{BMI}\left(\mathrm{kg} / \mathrm{m}^{2}\right)$ & $0.96(0.87,1.05)$ & 0.377 & $1.04(0.94,1.16)$ & 0.417 \\
\hline \multicolumn{5}{|l|}{$\begin{array}{l}\text { Gender [Female] } \\
\text { - Male }\end{array}$} \\
\hline Low back pain frequency & $0.85(0.70,1.02)$ & 0.103 & $0.77(0.64,0.94)$ & $0.009^{*}$ \\
\hline Load (Newtons) & & & $0.90(0.89,0.91)$ & $<0.001^{*}$ \\
\hline \multicolumn{5}{|l|}{ Smoking Status [Never smoked] } \\
\hline - Current smoker & $0.15(0.02,1.48)$ & 0.087 & $0.11(0.01,1.63)$ & 0.108 \\
\hline - Past smoker & $0.86(0.32,2.31)$ & 0.762 & $0.83(0.27,2.56)$ & 0.740 \\
\hline
\end{tabular}

* = significant, $B M I$ Body mass index, $\mathrm{kg}$ kilograms, $m$ metres

began to decrease with loading $\geq 30 \mathrm{~N}$, which is considerably lower than the values suggested by Kumar and Stoll. It may be the case that the rolling indenter head is less tolerable at higher loads when compared to indenter heads that move in a single plane only. In addition, participants who had experienced one or more days of lower back pain in the past week ( $77.3 \%$ of the sample) were also more likely to give a lower comfort rating compared to asymptomatic individuals. Therefore, while participants may report discomfort with increasing load or a history of back pain, we could not determine if the discomfort was from the test itself, was from provocation of sub-clinical conditions or was due to excessive loading. As tolerance for this type of testing appears to be individual in nature, we recommend that testing be performed to the participant's onset of discomfort rather than an absolute loading value.

The nature of the sensation produced by the rolling indenter head on the trunk was an important factor governing a participant's subjective experience of the assessment. This rolling action of the VerteTrack is novel when compared to the other mechanised tools for measuring spinal stiffness. The two wheels that form the rolling indenter head were 3D printed and made from Acrylonitrile butadiene styrene (ABS) plastic making them rigid and inflexible. Other non-rolling devices described in the literature have employed padded indenter heads $[9-12,20]$. It is likely that using a different material and/or providing some form of padding or lubricant for the device-subject interface may improve comfort. Any padding that is employed however, has the potential to contribute to measurement error through compression/deformation. It is therefore important that accuracy is not sacrificed in the quest for comfort. In their study, Lee and Evans [10] employed talcum powder in an attempt to reduce friction between the indenter head and the skin of the participants. In this research the indenter head was programmed to roll in a caudal to cephalad direction which may have caused discomfort and/or skin irritation as the indenter head moved 'uphill' from the apex of the lumbar lordosis to the lift-off point in the thoracolumbar junction. As the VerteTrack device can also be programmed to move in a cephalad to caudal direction it would be worthwhile establishing which direction or combination of directions is ideal for testing.

With regards to the time taken to perform the assessment, this research highlights that the lumbar trunk stiffness of a young adult can be assessed efficiently (mean assessment time of $11.6 \mathrm{~min}$ ) using the VerteTrack device. Allison et al. [21] investigated the influence of varied load orientation on lumbar spine stiffness in 24 normal subjects using the spinal P-A mobilization (SPAM) apparatus. These researchers reported that it took approximately $30 \mathrm{~min}$ to perform 12 measurements of the lumbar spine (L5, L3 and L1) for each participant at loads between 30 and $100 \mathrm{~N}$. Lee and Evans [10] measured the influence of loading rate on spinal stiffness at L3, L4 and L5 in a group of young adults. These authors reported that their assessment, including varied loading rates at three segment levels, took approximately $2 \mathrm{~h}$ to complete. While other researchers $[9,11-13]$ have utilised similar mechanical indentation devices for measuring spinal stiffness there is lack of data on the overall time taken to perform these assessments. It is important to note that the assessment time data in this study were skewed by a number of instances of software failure. The version of the software used in this study represented version 2.0. Future iterations of the software associated with the VerteTrack will lead to fewer software bugs and therefore shorter assessment times. Still, the time taken to test participants in this study highlights a significant improvement from prior devices, making the VerteTrack device suitable for use in clinical trials. 
There are several limitations associated with this study. Firstly, the spinal stiffness assessments were performed on a convenience sample made up of young adults (median age 23 years, range $=18-38$ years) from a tertiary-level student population. It is not clear how safe, comfortable or efficient this device would be for use in a paediatric or geriatric population. Furthermore, the sample was comprised of people from a general (non-clinical) population, which given the high prevalence of back pain in society included participants with and without varying levels of low back discomfort. The suitability of the VerteTrack device remains unclear in clinical research or clinical practice settings.

Interestingly, there was a higher than expected prevalence of LBP in our sample population. This may have been due to the fact that sample consisted entirely of chiropractic students studying in the postgraduate and undergraduate programs at Macquarie University. It is known that chiropractic students suffer from physical side-effects, e.g. lower back pain, from performing [22, 23] and/or receiving [24] spinal manipulative procedures during their training which may have contributed to acute cases of lower back pain in the sample. Participation in the study was entirely voluntary, however it is possible that the students that elected to participate were those who were either experiencing an acute low back pain episode or had a history of lower back pain. With the spine being a strong focus in chiropractic education, students in the program may demonstrate a hypersensitivity to spinal dysfunction which may have skewed the prevalence figures for lower back pain in this sample.

The terminal stiffness values and the variation between the various spinal levels obtained in this study are smaller than those observed in previous studies. Due to the action of the indenter roller it is likely that the displacement values obtained with the VerteTrack differ when compared to other devices as a function of time under load. The indenter roller moves quickly over the trunk and applies P-A loads up to a maximum of $60 \mathrm{~N}$. Both the rate of loading and magnitude of loading are unique in this study making comparisons between the terminal stiffness values obtained by previous devices and the VerteTrack problematic. This highlights the need to better understand the nuances involved with the interpretation of the trunk stiffness values obtained with this device.

Harms data were collected actively at the time of the trunk stiffness assessment and there was no planned active or passive harms surveillance post-assessment. Latent adverse reactions may have been captured more thoroughly via the inclusion of a dedicated follow-up period, but attribution of these events to testing is difficult.

In this study, there was a negative correlation between comfort ratings and loading. Loads were applied sequentially to the lumbar spine of a participant with a brief rest period between traces. A more robust determination of the comfort associated with the assessment may have been obtained by using a Latin square design, similar to the work by Edgecombe et al. [25], to assign loads in a random sequence.

As this form of assessment provides clinical data that is used alongside other diagnostic information to guide management decisions, it is of crucial importance that the reliability and accuracy of any mechanical indentation device be tested. Further research should also be performed in a variety of trunk regions in clinical populations of varying ages to determine the true value of the VerteTrack device in these scenarios.

\section{Conclusions}

Based on the findings of this study, it appears that the VerteTrack is well-tolerated by participants and can be used safely and efficiently to measure the P-A stiffness of the lower trunk in young adults. The device is strictly for research purposes at this time, however the ultimate suitability of the VerteTrack for use in research and clinical settings may be determined once data from this study is combined with data regarding the reliability and accuracy of the device.

\section{Additional file}

Additional file 1: VerteTrack being used on a model. (mov $58240 \mathrm{~kb}$ )

\section{Abbreviations}

BMI: Body mass index; cm: Centimetres; FRC: Functional residual capacity; kg: Kilograms; mm: Millimetres; N: Newtons; N/mm: Newtons per millimetre; P-A: Posterior to anterior; SS: Spinal stiffness

\section{Acknowledgements}

The authors would like to thank Damian Da Silva, Rafael Fabrega Teixiera and Madeline Morrison for their help with the data collection process.

Funding

There was no funding for this project.

Availability of data and materials

The de-identified datasets used and/or analysed during the current study are available from the corresponding author on reasonable request.

\section{Authors' contributions}

MS, AD, BTB and GK conceived and designed the study. BTB wrote and compiled the manuscript. $A B, V C, P L G, A D$, and $M S$ were involved with the data collection and/or analysis, and helped to edit and review the final manuscript. All authors read and approved the final manuscript.

Ethics approval and consent to participate

Ethics approval for this study was obtained on the 24th of April, 2016 from the Macquarie University human ethics committee - Reference number: 5,201,600,008. All participants consented to participation in this research study.

\section{Consent for publication}

The volunteer subject portrayed in the Vertetrack videos and images gave their consent for these items to be included in the manuscript. 


\section{Competing interests}

GK is the creator and developer of the VerteTrack device used in this research. GK was not involved with the data collection or analysis phase of this study. The authors declare that they have no competing interests.

\section{Publisher's Note}

Springer Nature remains neutral with regard to jurisdictional claims in published maps and institutional affiliations.

\section{Author details}

'Department of Chiropractic, Macquarie University, Balaclava Rd, North Ryde, NSW 2109, Australia. ${ }^{2}$ Department of Statistics, Macquarie University, Balaclava Rd, North Ryde 2109, NSW, Australia. ${ }^{3}$ Department of Physical Therapy, University of Alberta, Edmonton, Canada.

Received: 22 February 2017 Accepted: 20 July 2017

\section{Published online: 03 August 2017}

\section{References}

1. Binkley J, Stratford PW, Gill C. Interrater reliability of lumbar accessory motion mobility testing. Phys Ther. 1995;75(9):786-95.

2. Matyas TA, Bach TM. The reliability of selected techniques in clinical arthrometrics. Aust J Physiother. 1985:31(5):175-99.

3. Maltland G. Vertebral manipulation. 4th ed. London: Butterworth Pty Ltd; 1977

4. Latimer J, Lee M, Adams RD. The effects of high and low loading forces on measured values of lumbar stiffness. J Manip Physiol Ther. 1998;21(3):157-63.

5. Nicholson LL, Adams RD, Maher CG. Manual discrimination capability when only viscosity is varied in viscoelastic stiffness stimuli. J Manip Physiol Ther. 2003:26(6):365-73.

6. Tuttle N. Is it reasonable to use an individual Patient's progress after treatment as a guide to ongoing clinical reasoning? J Manip Physiol Ther. 2009;32(5):396-403.

7. Phillips DR, Twomey LT. A comparison of manual diagnosis with a diagnosis established by a uni-level lumbar spinal block procedure. Manual Ther 1996;1(2):82-7.

8. Maher CG, Adams R, Shields RK. Reliability of pain and stiffness assessments in clinical manual lumbar spine examination. Phys Ther. 1994:74(9):801-11.

9. Lee $M$, Svensson NL. Measurement of stiffness during simulated spinal physiotherapy. Clin Phys Physiol M. 1990;11(3):201-7.

10. Lee RYW, Evans J. Load-displacement-time characteristics of the spine under posteroanterior mobilisation. Aust J Physiother. 1992;38(2):115-23.

11. Kumar S, Stoll S. Device, protocol and measurement of regional spinal stiffness. J Electromyogr Kines. 2011;21(3):458-65

12. Edmondston SJ, Allison GT, Gregg CD, Purden SM, Svansson GR, Watson AE. Effect of position on the posteroanterior stiffness of the lumbar spine. Manual Ther. 1998:3(1):21-6.

13. Fritz JM, Koppenhaver SL, Kawchuk GN, Teyhen DS, Hebert JJ, Childs JD. Preliminary investigation of the mechanisms underlying the effects of manipulation: exploration of a multi-variate model including spinal stiffness, multifidus recruitment, and clinical findings. Spine. 2011;36(21):1772-81.

14. Kawchuk G, Herzog W. A new technique of tissue stiffness (compliance) assessment: its reliability, accuracy and comparison with an existing method. J Manip Physiol Ther. 1996;19(1):13-8.

15. Latimer J, Lee M, Goodsell M, Maher CG, Wilkinson B, Adams R. Instrumented measurement of spinal stiffness. Manual Ther. 1996:1(4):204-9.

16. Hernandez L, Alhemood A, Genaidy AM, Karwowski W. Evaluation of different scales for measurement of perceived physical strain during performance of manual tasks. Int J Occu Saf Ergo. 2002;8(4):413-32.

17. Wong AY, Kawchuk G, Parent E, Prasad N. Within- and between-day reliability of spinal stiffness measurements obtained using a computer controlled mechanical indenter in individuals with and without low back pain. Man Ther. 2013;18(5):395-402.

18. Merz OWU, Robert M, Gesing $\vee$, Rominger M. Validity of palpation techniques for the identification of the spinous process L5. Manual Ther 2013;18(4):333-8.

19. Hsieh HF, Shannon SE. Three approaches to qualitative content analysis. Qual Health Res. 2005;15(9):1277-88.

20. Kumar S. Spinal stiffness in asymptomatic subjects. J Electromyogr Kines. 2011;21(5):762-6.
21. Allison GT, Edmondston SJ, Roe CP, Reid SE, Toy DA, Lundgren HE. Influence of load orientation on the posteroanterior stiffness of the lumbar spine. J Manip Physiol Ther. 1998;21(8):534-8.

22. Bisiacchi DW, Huber LL. Physical injury assessment of male versus female chiropractic students when learning and performing various adjustive techniques: a preliminary investigate study. Chiropr Osteopat. 2006;14:17.

23. Ndetan HT, Rupert RL, Bae S, Singh KP. Prevalence of musculoskeletal injuries sustained by students while attending a chiropractic college. J Manipulative Physiol Thera. 2009;32(2):140-8.

24. Macanuel K, Deconinck A, Sloma K, LeDoux M, Gleberzon BJ. Characterization of side effects sustained by chiropractic students during their undergraduate training in technique class at a chiropractic college: a preliminary retrospective study. J Can Chiropr Assoc. 2005;49(1):46-55.

25. Edgecombe TL, Kawchuk GN, Long CR, Pickar JG. The effect of application site of spinal manipulative therapy (SMT) on spinal stiffness. Spine J. 2015; 15(6):1332-8.

\section{Submit your next manuscript to BioMed Central and we will help you at every step:}

- We accept pre-submission inquiries

- Our selector tool helps you to find the most relevant journal

- We provide round the clock customer support

- Convenient online submission

- Thorough peer review

- Inclusion in PubMed and all major indexing services

- Maximum visibility for your research

Submit your manuscript at www.biomedcentral.com/submit 